\title{
Search for Charged Higgs Bosons Using the OPAL Detector at LEP
}

\author{
The OPAL Collaboration
}

\begin{abstract}
A search is described to detect charged Higgs bosons via the process $\mathrm{Z}^{0} \rightarrow \mathrm{H}^{+} \mathrm{H}^{-}$, using data collected by the OPAL detector at LEP which correspond to an integrated luminosity of approximately $110 \mathrm{pb}^{-1}$. It is assumed that the $\mathrm{H}^{+}$boson decays only to $\tau^{+} \nu_{\tau}$ and $\mathrm{c} \overline{\mathrm{s}}$ final states. From the negative outcome of this search a lower bound of $44.1 \mathrm{GeV}$ (95\% CL) is derived for the mass of the charged Higgs boson.
\end{abstract}

(Submitted to Physics Letters B) 


\section{The OPAL Collaboration}

G. Alexander ${ }^{23}$, J. Allison ${ }^{16}$, N. Altekamp ${ }^{5}$, K. Ametewee ${ }^{25}$, K.J. Anderson ${ }^{9}$, S. Anderson ${ }^{12}$, S. Arcelli ${ }^{2}$, S. Asai ${ }^{24}$, D. Axen ${ }^{29}$, G. Azuelos ${ }^{18, a}$, A.H. Ball ${ }^{17}$, E. Barberio ${ }^{26}$, R.J. Barlow ${ }^{16}$, R. Bartoldus ${ }^{3}$, J.R. Batley ${ }^{5}$, G. Beaudoin ${ }^{18}$, J. Bechtluft ${ }^{14}$, G.A. Beck ${ }^{13}$, C. Beeston ${ }^{16}$, T. Behnke ${ }^{8}$, A.N.Bell ${ }^{1}$, K.W. Bell ${ }^{20}$, G. Bella ${ }^{23}$, S. Bentvelsen ${ }^{8}$, P. Berlich ${ }^{10}$, S. Bethke $^{14}$, O. Biebel ${ }^{14}$, I.J. Bloodworth ${ }^{1}$, J.E. Bloomer ${ }^{1}$, P. Bock ${ }^{11}$, H.M. Bosch ${ }^{11}$, M. Boutemeur ${ }^{18}$, B.T. Bouwens ${ }^{12}$, S. Braibant ${ }^{12}$, P. Bright-Thomas ${ }^{25}$, R.M. Brown ${ }^{20}$, H.J. Burckhart ${ }^{8}$,

C. Burgard ${ }^{27}$, R. Bürgin ${ }^{10}$, P. Capiluppi ${ }^{2}$, R.K. Carnegie ${ }^{6}$, A.A. Carter $^{13}$, J.R. Carter ${ }^{5}$, C.Y.Chang ${ }^{17}$, C. Charlesworth ${ }^{6}$, D.G. Charlton ${ }^{1, b}$, D. Chrisman ${ }^{4}$, S.L. Chu ${ }^{4}$, P.E.L. Clarke ${ }^{15}$, S.G. Clowes ${ }^{16}$, I. Cohen ${ }^{23}$, J.E. Conboy ${ }^{15}$, O.C. Cooke ${ }^{16}$, M. Cuffiani ${ }^{2}$, S. Dado ${ }^{22}$,

C. Dallapiccola ${ }^{17}$, G.M. Dallavalle ${ }^{2}$, C.Darling ${ }^{31}$, S. De Jong ${ }^{12}$, L.A. del Pozo ${ }^{8}$, M.S. Dixit ${ }^{7}$, E. do Couto e Silva ${ }^{12}$, E. Duchovni ${ }^{26}$, G. Duckeck ${ }^{8}$, I.P. Duerdoth ${ }^{16}$, U.C. Dunwoody ${ }^{8}$, J.E.G. Edwards ${ }^{16}$, P.G. Estabrooks ${ }^{6}$, H.G. Evans ${ }^{9}$, F. Fabbri ${ }^{2}$, B. Fabbro ${ }^{21}$, P. Fath ${ }^{11}$, F. Fiedler ${ }^{12}$, M. Fierro ${ }^{2}$, M. Fincke-Keeler ${ }^{28}$, H.M. Fischer ${ }^{3}$, R. Folman ${ }^{26}$, D.G. Fong ${ }^{17}$, M. Foucher ${ }^{17}$, H. Fukui ${ }^{24}$, A. Fürtjes ${ }^{8}$, P. Gagnon ${ }^{7}$, A. Gaidot ${ }^{21}$, J.W. Gary ${ }^{4}$, J. Gascon ${ }^{18}$, S.M. Gascon-Shotkin ${ }^{17}$, N.I. Geddes ${ }^{20}$, C. Geich-Gimbel ${ }^{3}$, S.W. Gensler ${ }^{9}$, F.X. Gentit ${ }^{21}$, T. Geralis ${ }^{20}$, G. Giacomelli ${ }^{2}$, P. Giacomelli ${ }^{4}$, R. Giacomelli ${ }^{2}$, V. Gibson ${ }^{5}$, W.R. Gibson ${ }^{13}$,

D.M. Gingrich ${ }^{30, a}$, J. Goldberg ${ }^{22}$, M.J. Goodrick ${ }^{5}$, W. Gorn ${ }^{4}$, C. Grandi ${ }^{2}$, E. Gross ${ }^{26}$, C. Hajdu ${ }^{32}$, G.G.Hanson ${ }^{12}$, M. Hansroul ${ }^{8}$, M.Hapke ${ }^{13}$, C.K.Hargrove ${ }^{7}$, P.A. Hart ${ }^{9}$, C. Hartmann ${ }^{3}$, M. Hauschild ${ }^{8}$, C.M. Hawkes ${ }^{8}$, R. Hawkings ${ }^{8}$, R.J. Hemingway ${ }^{6}$, G. Herten ${ }^{10}$, R.D. Heuer ${ }^{8}$, M.D. Hildreth ${ }^{8}$, J.C. Hill ${ }^{5}$, S.J. Hillier ${ }^{8}$, T. Hilse ${ }^{10}$, P.R.Hobson ${ }^{25}$, D. Hochman ${ }^{26}$, R.J. Homer ${ }^{1}$, A.K. Honma ${ }^{28, a}$, D. Horváth ${ }^{32, c}$, R. Howard ${ }^{29}$, R.E. Hughes-Jones ${ }^{16}$, D.E. Hutchcroft ${ }^{5}$, P. Igo-Kemenes ${ }^{11}$, D.C. Imrie ${ }^{25}$, A. Jawahery ${ }^{17}$, P.W. Jeffreys ${ }^{20}$, H. Jeremie ${ }^{18}$, M. Jimack ${ }^{1}$, A. Joly ${ }^{18}$, M. Jones ${ }^{6}$, R.W.L. Jones ${ }^{8}$, U. Jost ${ }^{11}$, P. Jovanovic ${ }^{1}$, D. Karlen ${ }^{6}$, T. Kawamoto ${ }^{24}$, R.K. Keeler ${ }^{28}$, R.G. Kellogg ${ }^{17}$, B.W. Kennedy ${ }^{20}$, B.J. King ${ }^{8}$, J. King ${ }^{13}$, J. Kirk ${ }^{29}$, S. Kluth ${ }^{5}$, T. Kobayashi ${ }^{24}$, M. Kobel ${ }^{10}$, D.S. Koetke ${ }^{6}$, T.P. Kokott ${ }^{3}$, S. Komamiya ${ }^{24}$, R. Kowalewski ${ }^{8}$, T. Kress ${ }^{11}$, P. Krieger ${ }^{6}$, J. von Krogh ${ }^{11}$, P. Kyberd ${ }^{13}$, G.D. Lafferty ${ }^{16}$, H. Lafoux ${ }^{21}$, R. Lahmann ${ }^{17}$, W.P. Lai ${ }^{19}$, D. Lanske ${ }^{14}$, J. Lauber ${ }^{15}$, J.G. Layter ${ }^{4}$, A.M. Lee ${ }^{31}$, E. Lefebvre ${ }^{18}$, D. Lellouch ${ }^{26}$, J. Letts ${ }^{2}$, L. Levinson ${ }^{26}$, C. Lewis ${ }^{15}$, S.L. Lloyd ${ }^{13}$, F.K. Loebinger ${ }^{16}$, G.D. Long ${ }^{17}$, B. Lorazo ${ }^{18}$, M.J. Losty ${ }^{7}$, J. Ludwig ${ }^{10}$, A. Luig ${ }^{10}$, A. Malik ${ }^{21}$, M. Mannelli ${ }^{8}$, S. Marcellini ${ }^{2}$, C. Markus ${ }^{3}$, A.J. Martin ${ }^{13}$, J.P. Martin ${ }^{18}$, G. Martinez ${ }^{17}$, T. Mashimo ${ }^{24}$, W. Matthews ${ }^{25}$, P. Mättig ${ }^{3}$, W.J. McDonald ${ }^{30}$, J. McKenna ${ }^{29}$, E.A. Mckigney ${ }^{15}$, T.J.McMahon ${ }^{1}$, A.I. McNab ${ }^{13}$, F. Meijers ${ }^{8}$, S. Menke ${ }^{3}$, F.S. Merritt ${ }^{9}$, H. Mes ${ }^{7}$, J. Meyer ${ }^{27}$, A. Michelini ${ }^{8}$, G. Mikenberg ${ }^{26}$, D.J. Miller ${ }^{15}$, R. Mir ${ }^{26}$, W. Mohr ${ }^{10}$, A.Montanari ${ }^{2}$, T. Mori ${ }^{24}$, M. Morii ${ }^{24}$, U. Müller ${ }^{3}$, B. Nellen ${ }^{3}$, B. Nijjhar ${ }^{16}$, R. Nisius ${ }^{8}$, S.W. O'Neale ${ }^{1}$, F.G. Oakham ${ }^{7}$, F. Odorici ${ }^{2}$, H.O. Ogren ${ }^{12}$, N.J. Oldershaw ${ }^{16}$, T. Omori ${ }^{24}$, C.J. Oram ${ }^{28, a}$, M.J. Oreglia ${ }^{9}$, S. Orito $^{24}$, M.Palazzo ${ }^{2}$, J.Pálinkás ${ }^{33}$, F.M.Palmonari ${ }^{2}$, J.P. Pansart ${ }^{21}$, G. Pásztor ${ }^{32}$, J.R. Pater $^{16}$, G.N.Patrick ${ }^{20}$, M.J. Pearce ${ }^{1}$, P.D.Phillips ${ }^{16}$, J.E. Pilcher ${ }^{9}$, J. Pinfold ${ }^{30}$, D.E. Plane ${ }^{8}$, P. Poffenberger ${ }^{28}$, B. Poli ${ }^{2}$, A. Posthaus ${ }^{3}$, T.W. Pritchard ${ }^{13}$, H. Przysiezniak ${ }^{30}$, D.L. Rees ${ }^{1}$, D. Rigby ${ }^{1}$, M.G. Rison ${ }^{5}$, S.A. Robins ${ }^{13}$, N. Rodning ${ }^{30}$, J.M. Roney $^{28}$, E. Ros $^{8}$,

A.M. Rossi ${ }^{2}$, M. Rosvick ${ }^{28}$, P. Routenburg ${ }^{30}, Y_{\text {. Rozen }}{ }^{8}$, K. Runge $^{10}$, O. Runolfsson ${ }^{8}$, D.R.Rust ${ }^{12}$, R. Rylko ${ }^{25}$, E.K.G. Sarkisyan ${ }^{23}$, M.Sasaki ${ }^{24}$, C.Sbarra ${ }^{2}$, A.D.Schaile ${ }^{8}$, O.Schaile ${ }^{10}$, F.Scharf ${ }^{3}$, P.Scharff-Hansen ${ }^{8}$, P.Schenk ${ }^{4}$, B.Schmitt ${ }^{3}$, M.Schröder ${ }^{8}$, H.C.Schultz-Coulon ${ }^{10}$, M.Schulz ${ }^{8}$, P.Schütz ${ }^{3}$, J.Schwiening ${ }^{3}$, W.G.Scott ${ }^{20}$, T.G.Shears ${ }^{16}$, 
B.C.Shen ${ }^{4}$, C.H.Shepherd-Themistocleous ${ }^{27}$, P. Sherwood ${ }^{15}$, G.P. Siroli ${ }^{2}$, A.Sittler ${ }^{27}$, A.Skillman ${ }^{15}$, A.Skuja ${ }^{17}$, A.M.Smith ${ }^{8}$, T.J.Smith ${ }^{28}$, G.A.Snow ${ }^{17}$, R. Sobie ${ }^{28}$, S. Söldner-Rembold ${ }^{10}$, R.W.Springer ${ }^{30}$, M.Sproston ${ }^{20}$, A.Stahl ${ }^{3}$, M.Starks ${ }^{12}$, C.Stegmann ${ }^{10}$,

K. Stephens ${ }^{16}$, J.Steuerer ${ }^{28}$, B.Stockhausen ${ }^{3}$, D. Strom ${ }^{19}$, F.Strumia ${ }^{8}$, P.Szymanski ${ }^{20}$,

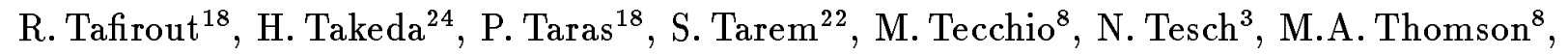
E. von Törne ${ }^{3}$, S. Towers ${ }^{6}$, M. Tscheulin ${ }^{10}$, T. Tsukamoto ${ }^{24}$, E. Tsur ${ }^{23}$, A.S. Turcot ${ }^{9}$, M.F. Turner-Watson ${ }^{8}$, P. Utzat ${ }^{11}$, R. Van Kooten ${ }^{12}$, G. Vasseur ${ }^{21}$, P. Vikas ${ }^{18}$, M. Vincter ${ }^{28}$, E.H. Vokurka ${ }^{16}$, F. Wäckerle ${ }^{10}$, A. Wagner ${ }^{27}$, D.L. Wagner ${ }^{9}$, C.P. Ward ${ }^{5}$, D.R. Ward ${ }^{5}$, J.J. Ward ${ }^{15}$, P.M. Watkins ${ }^{1}$, A.T. Watson ${ }^{1}$, N.K. Watson ${ }^{7}$, P. Weber ${ }^{6}$, P.S. Wells ${ }^{8}$, N. Wermes ${ }^{3}$, B. Wilkens ${ }^{10}$, G.W.Wilson ${ }^{27}$, J.A.Wilson ${ }^{1}$, T. Wlodek ${ }^{26}$, G. Wolf ${ }^{26}$, S. Wotton ${ }^{11}$, T.R. Wyatt ${ }^{16}$, S. Xella ${ }^{2}$, S. Yamashita ${ }^{24}$, G. Yekutieli ${ }^{26}$, V. Zacek ${ }^{18}$,

${ }^{1}$ School of Physics and Space Research, University of Birmingham, Birmingham B15 2TT, UK ${ }^{2}$ Dipartimento di Fisica dell' Università di Bologna and INFN, I-40126 Bologna, Italy

${ }^{3}$ Physikalisches Institut, Universität Bonn, D-53115 Bonn, Germany

${ }^{4}$ Department of Physics, University of California, Riverside CA 92521, USA

${ }^{5}$ Cavendish Laboratory, Cambridge CB3 0HE, UK

${ }^{6}$ Ottawa-Carleton Institute for Physics, Department of Physics, Carleton University, Ottawa, Ontario K1S 5B6, Canada

${ }^{7}$ Centre for Research in Particle Physics, Carleton University, Ottawa, Ontario K1S 5B6, Canada

${ }^{8}$ CERN, European Organisation for Particle Physics, CH-1211 Geneva 23, Switzerland

${ }^{9}$ Enrico Fermi Institute and Department of Physics, University of Chicago, Chicago IL 60637, USA

${ }^{10}$ Fakultät für Physik, Albert Ludwigs Universität, D-79104 Freiburg, Germany

${ }^{11}$ Physikalisches Institut, Universität Heidelberg, D-69120 Heidelberg, Germany

${ }^{12}$ Indiana University, Department of Physics, Swain Hall West 117, Bloomington IN 47405, USA

${ }^{13}$ Queen Mary and Westfield College, University of London, London E1 4NS, UK

${ }^{14}$ Technische Hochschule Aachen, III Physikalisches Institut, Sommerfeldstrasse 26-28, D-52056

Aachen, Germany

${ }^{15}$ University College London, London WC1E 6BT, UK

${ }^{16}$ Department of Physics, Schuster Laboratory, The University, Manchester M13 9PL, UK

${ }^{17}$ Department of Physics, University of Maryland, College Park, MD 20742, USA

${ }^{18}$ Laboratoire de Physique Nucléaire, Université de Montréal, Montréal, Quebec H3C 3J7, Canada

${ }^{19}$ University of Oregon, Department of Physics, Eugene OR 97403, USA

${ }^{20}$ Rutherford Appleton Laboratory, Chilton, Didcot, Oxfordshire OX11 0QX, UK

${ }^{21}$ CEA, DAPNIA/SPP, CE-Saclay, F-91191 Gif-sur-Yvette, France

${ }^{22}$ Department of Physics, Technion-Israel Institute of Technology, Haifa 32000, Israel

${ }^{23}$ Department of Physics and Astronomy, Tel Aviv University, Tel Aviv 69978, Israel

${ }^{24}$ International Centre for Elementary Particle Physics and Department of Physics, University of Tokyo, Tokyo 113, and Kobe University, Kobe 657, Japan

${ }^{25}$ Brunel University, Uxbridge, Middlesex UB8 3PH, UK

${ }^{26}$ Particle Physics Department, Weizmann Institute of Science, Rehovot 76100, Israel 
${ }^{27}$ Universität Hamburg/DESY, II Institut für Experimental Physik, Notkestrasse 85, D-22607 Hamburg, Germany

${ }^{28}$ University of Victoria, Department of Physics, P O Box 3055, Victoria BC V8W 3P6, Canada ${ }^{29}$ University of British Columbia, Department of Physics, Vancouver BC V6T 1Z1, Canada

${ }^{30}$ University of Alberta, Department of Physics, Edmonton AB T6G 2J1, Canada

${ }^{31}$ Duke University, Dept of Physics, Durham, NC 27708-0305, USA

${ }^{32}$ Research Institute for Particle and Nuclear Physics, H-1525 Budapest, P O Box 49, Hungary

${ }^{33}$ Institute of Nuclear Research, H-4001 Debrecen, P O Box 51, Hungary

${ }^{a}$ Also at TRIUMF, Vancouver, Canada V6T $2 \mathrm{~A} 3$

${ }^{b}$ Royal Society University Research Fellow

${ }^{c}$ Institute of Nuclear Research, Debrecen, Hungary 


\section{Introduction}

The interactions between elementary particles are well described by the Standard Model (SM) [1]. However, basic questions such as the mechanism of spontaneous symmetry breaking and the origin of mass are still awaiting a satisfactory explanation. The Higgs mechanism [2] proposes possible answers but, lacking the material proof that would be provided by the discovery of a Higgs boson, the proposed mechanism remains an attractive working hypothesis only.

In the minimal SM, which uses one scalar field doublet, a single neutral Higgs boson is predicted. Extensions of the model predict a larger Higgs sector [3]. The minimal extension of the SM, which uses two scalar field doublets, predicts five Higgs bosons of which three are neutral $\left(\mathrm{h}^{0}, \mathrm{H}^{0}\right.$ and $\left.\mathrm{A}^{0}\right)$ and two are charged $\left(\mathrm{H}^{+}\right.$and $\left.\mathrm{H}^{-}\right)$. The discovery of a charged Higgs boson would point without ambiguity towards a non-minimal Higgs sector. Beyond the SM, supersymmetric (SUSY) extensions are today regarded as the most promising models since they propose "natural" answers to the question of energy scales (the problem of hierarchy) and, at the same time, share the success of the SM in describing the observed particle physics phenomena. At the tree level, the Minimal Supersymmetric Extension of the Standard Model (MSSM), a SUSY model with two scalar field doublets, predicts for the mass, $m_{\mathrm{H}^{ \pm}}$, of the charged Higgs bosons $m_{\mathrm{H}^{ \pm}}>M_{\mathrm{W}^{ \pm}}$. This relation is modified only slightly when radiative loop corrections are considered. Consequently, the detection of the process $\mathrm{Z}^{0} \rightarrow \mathrm{H}^{+} \mathrm{H}^{-}$, which would imply $m_{\mathrm{H}^{ \pm}}<M_{\mathrm{Z}^{0}} / 2$, would invalidate the MSSM.

Higgs boson searches at the $\mathbf{e}^{+} \mathbf{e}^{-}$collider LEP have placed lower bounds on the mass of the SM Higgs boson $(\approx 60 \mathrm{GeV}[4])$ and, within the MSSM, on those of the scalar $h^{0}(\approx 44 \mathrm{GeV})$ and the pseudoscalar $A^{0}(\approx 24 \mathrm{GeV})[5]$. Searches for the process $\mathrm{Z}^{0} \rightarrow \mathrm{H}^{+} \mathrm{H}^{-}$have also been carried out [6] [7] [8]. The best limit so far is $m_{\mathrm{H}^{ \pm}}>43.5 \mathrm{GeV}$ (95\% CL) [7]. In a previous search based on about 30,000 $\mathrm{Z}^{0}$ decays, OPAL obtained $m_{\mathrm{H}^{ \pm}}>35 \mathrm{GeV}(95 \% \mathrm{CL})$ [6]. The present work describes a new analysis based on more than 4 million $\mathrm{Z}^{0}$ decays.

The decay width of the process $\mathrm{e}^{+} \mathrm{e}^{-} \rightarrow \mathrm{Z}^{0} \rightarrow \mathrm{H}^{+} \mathrm{H}^{-}$is given by [9]:

$$
\Gamma\left(\mathrm{Z}^{0} \rightarrow \mathrm{H}^{+} \mathrm{H}^{-}\right)=\frac{G_{F} M_{\mathrm{Z}^{0}}^{3}}{6 \sqrt{2} \pi}\left(\frac{1}{2}-\sin ^{2} \theta_{W}\right)^{2}\left(1-\frac{4 m_{\mathrm{H}^{ \pm}}^{2}}{s}\right)^{3 / 2}
$$

where $G_{F}$ is the weak coupling constant and $\theta_{W}$ the electroweak mixing angle. At a centre of mass energy $\sqrt{s}=M_{\mathrm{Z}^{0}}$ and for $m_{\mathrm{H}^{ \pm}}=40 \mathrm{GeV}$ this yields a cross section of $46 \mathrm{pb}$. For $M_{\tau}<m_{\mathrm{H}^{ \pm}}<\left(M_{\mathrm{t}}+M_{\mathrm{b}}\right)$ the charged Higgs boson decays preferentially into $\tau^{+} \nu_{\tau}$ and $\bar{c}$. The $\mathrm{H}^{+} \rightarrow \mathrm{c} \overline{\mathrm{b}}$ decay is suppressed by the small element, $V_{c b}$, of the CKM matrix. The decay of the charged Higgs boson is model dependent; although in models with two field doublets one can assume that the two main channels, $\tau^{+} \nu_{\tau}$ and $c \bar{s}$, saturate the decay [3].

The present search is carried out separately for three possible final states: leptonic, $\mathrm{H}^{+} \mathrm{H}^{-} \rightarrow$ $\left(\tau^{+} \nu_{\tau}\right)\left(\tau^{-} \bar{\nu}_{\tau}\right)$; semi-leptonic, $\mathrm{H}^{+} \mathrm{H}^{-} \rightarrow\left(\tau^{+} \nu_{\tau}\right)(\overline{\mathrm{c}} \mathrm{s})+\left(\tau^{-} \bar{\nu}_{\tau}\right)(\mathrm{c} \overline{\mathrm{s}})$; hadronic, $\mathrm{H}^{+} \mathrm{H}^{-} \rightarrow(\mathrm{cs})(\overline{\mathrm{c}} \mathrm{s})$. In a sample of $N_{\mathrm{Z}^{0}}(\sqrt{s}) \mathrm{Z}^{0}$ decays taken at fixed $\sqrt{s}$, the expected number of $\mathrm{H}^{+} \mathrm{H}^{-}$events with the Higgs bosons decaying into final states $i$ and $j$ is given by

$$
N_{i j}(\sqrt{s})=N_{\mathrm{Z}^{0}}(\sqrt{s}) \frac{\Gamma\left(\mathrm{Z}^{0} \rightarrow \mathrm{H}^{+} \mathrm{H}^{-}\right)}{\Gamma_{\mathrm{Z}^{0}}} B r_{i} B r_{j} \epsilon_{i j}
$$

where $\Gamma_{\mathrm{Z}^{0}}$ is the width of the $\mathrm{Z}^{0}$ boson, $B r_{i, j}$ are the $\mathrm{H}^{ \pm}$branching ratios and $\epsilon_{i j}$ is the detection efficiency for the final state $\mathrm{H}^{+} \mathrm{H}^{-} \rightarrow i j$. 
The searches for the semi-leptonic and hadronic final states use selection criteria which do not depend explicitly on the assumed quark flavour but rather on kinematic distributions of the reconstructed hadron jets. Due to their harder fragmentation, $b$-jets are reconstructed more efficiently than those of light-flavoured quarks. For this reason, the results which are obtained for the $c \bar{s}$ final state can be assumed to be valid even in the case of sizeable contributions from $\mathrm{H}^{+} \rightarrow \mathrm{c} \bar{b}$. By combining the searches in the leptonic, semi-leptonic and hadronic channels, mass limits are obtained for any value of the branching ratio $B r\left(\mathrm{H}^{+} \rightarrow \tau^{+} \nu_{\tau}\right)$.

\section{Experimental data}

The present search is based on data collected by the OPAL experiment up to the end of 1994 . It corresponds to an integrated luminosity of approximately $110 \mathrm{pb}^{-1}$, where $80 \%$ were collected at $\sqrt{s} \approx M_{\mathrm{Z}^{0}}, 10 \%$ at $\sqrt{s} \approx M_{\mathrm{Z}^{0}}-2 \mathrm{GeV}$ and $10 \%$ at $\sqrt{s} \approx M_{\mathrm{Z}^{0}}+2 \mathrm{GeV}$.

The OPAL detector [10], with its acceptance of nearly $4 \pi$ steradians, and with its good tracking, calorimetry and particle identification capabilities, is well suited to this analysis which searches for widely different event topologies. The apparatus consists of a central tracking detector inside a 0.435 Tesla magnetic field surrounded by a lead-glass electromagnetic calorimeter together with presamplers and time-of-flight scintillators which are located outside the magnet coil. The magnet return yoke is instrumented for hadron calorimetry and is covered by external muon chambers. Calorimeters close to the beam axis form the forward detector which measures the luminosity and completes the geometrical acceptance. The forward detector contains lead-scintillator calorimeters which were later complemented by silicon-tungsten calorimeters.

The event analysis uses charged particle tracks and electromagnetic energy clusters selected according to standard quality requirements [11] [12]. Accepted tracks have more than 20 measured space points, originate from the vicinity of the $\mathrm{e}^{+} \mathrm{e}^{-}$interaction point, and have a transverse momentum in excess of $50 \mathrm{MeV}$. Accepted electromagnetic clusters in the barrel region (with polar angle $\theta$ satisfying $|\cos \theta|<0.82$ ) have energies of greater than $100 \mathrm{MeV}$. Those in the endcap region $(0.81<|\cos \theta|<0.984)$ have more than $200 \mathrm{MeV}$ energy and consist of at least two adjacent lead glass blocks. Energy clusters in the hadron calorimeter are only used in the semi-leptonic channel to improve the hadronic mass resolution. In the hadronic final state the best mass resolution is obtained by kinematic fitting.

The search for the leptonic final state is based on those $\mathrm{Z}^{0}$ boson decays with low multiplicity where the number of tracks and clusters is limited to less than 19 in total. The sample contains mainly leptonic $\mathrm{Z}^{0}$ boson decays, two-photon events, low-multiplicity hadronic $\mathrm{Z}^{0}$ boson decays and cosmic ray muons. Cosmic ray muons are eliminated using the timing information from the time-of-flight counters and by checking the matching of the tracks to the $\mathrm{e}^{+} \mathrm{e}^{-}$interaction point. For the semi-leptonic and the hadronic final states the standard selection of hadronic $\mathrm{Z}^{0}$ decays [11] is used. This requires events to have at least 5 tracks and 7 clusters and a minimum energy in the electromagnetic calorimeter, $E / \sqrt{s}>0.1$. In all three search channels, two-photon events are reduced by requiring less than $2 \mathrm{GeV}$ energy in the forward detector.

The signal detection efficiencies and background suppression factors are obtained from extensive Monte Carlo simulations. For the $\mathrm{Z}^{0} \rightarrow \mathrm{H}^{+} \mathrm{H}^{-}$process the event generator embedded in the PYTHIA program package [13] is used. The process $\mathrm{Z}^{0} \rightarrow$ hadrons is generated using the JETSET 7.3 parton-shower Monte Carlo [14] with parameters tuned to OPAL data [15] 
which is also used to model the hadronization process in $\mathrm{H}^{ \pm}$decays. For $\mathrm{Z}^{0} \rightarrow \mu^{+} \mu^{-}$and $\tau^{+} \tau^{-}$, KORALZ [16] is used and for $\mathrm{Z}^{0} \rightarrow \mathbf{e}^{+} \mathbf{e}^{-}$, BABAMC [17]. The generated events are processed by the OPAL detector simulation [18] and event reconstruction programs. In general, the full simulation of the OPAL detector is used. In some cases a less detailed but faster version of the simulation is used to obtain higher statistics.

\subsection{The leptonic final state}

The signature for the leptonic channel, $\mathrm{H}^{+} \mathrm{H}^{-} \rightarrow\left(\tau^{+} \nu_{\tau}\right)\left(\tau^{-} \bar{\nu}_{\tau}\right)$, is a pair of low-multiplicity, acoplanar jets. Since the $\tau^{ \pm}$decay products are strongly collimated, a cone algorithm is used to recognize possible signal events. The cone algorithm starts with the particle (track or cluster) having the highest energy and searches within a cone of $20^{\circ}$ half-angle for the particle with the next-highest energy. If such a particle is found, the two momentum vectors are added to define the axis of a new cone and the procedure is repeated until no more particles can be added to that cone. Of the remaining particles, the particle with the highest energy is taken as the starting point to build a new cone, and the above procedure is repeated until all particles are assigned to a cone. The energy inside a cone is then calculated by adding up the energy of the particles. Special care is taken to avoid double-counting of energy in the case of charged particles for which the energy is measured both in the tracking detector and in the electromagnetic calorimeters. If one or several tracks are pointing towards a given cluster, the momentum-sum of the tracks is subtracted from the cluster energy, unless the momentumsum is larger than the cluster energy in which case the cluster energy is disregarded. The same procedure is also used to calculate the visible energy, $E_{v i s}$, of the entire event.

An event is retained if it contains exactly two cones with at least one charged particle per cone. The energy of each cone has to be larger than $2 \mathrm{GeV}$ and the polar angle of the two cone axes has to satisfy $|\cos \theta|<0.7$. The energy not included in the two cones has to be less than $1 \%$ of the beam energy. In addition, no charged track is allowed to occur outside the two cones and no cluster is allowed to occur close to the inner edge of the endcap electromagnetic calorimeter $(0.97<|\cos \theta|<0.984)$.

The background from $\mathrm{Z}^{0} \rightarrow \ell^{+} \ell^{-}$is eliminated by requiring the acoplanarity angle ${ }^{1}$, defined by the two cone axes, to be larger than $20^{\circ}$. To this end it is essential to measure the acoplanarity angle with good precision. When a charged particle with high transverse momentum, $p_{t}$, traverses the tracking detector close to a cathode wire plane, the track reconstruction algorithm occasionally fails to resolve the left-right ambiguity. This creates a spurious mirror track which biases the measurement of the acoplanarity angle. Therefore, events are eliminated if they contain a track with $p_{t}>5 \mathrm{GeV}$, with azimuthal angle within $1^{\circ}$ of that of a cathode plane and no associated electromagnetic cluster. This cut affects less than $0.1 \%$ of the events.

The scatter plot of the normalized visible energy vs. the acoplanarity angle of the two cone axes is shown in Fig. 1 for the data and for simulated $\mathrm{H}^{+} \mathrm{H}^{-}$events with $m_{\mathrm{H}^{ \pm}}=44 \mathrm{GeV}$. Events with small visible energy from two-photon processes are removed by the cut $E_{v i s} / \sqrt{s}>0.15$ indicated in the figure. The acoplanarity angle distribution for the data, after the cut, is shown in the insert. The tail towards large acoplanarity angles comes from $\tau^{+} \tau^{-}$events where one or

\footnotetext{
${ }^{1}$ The acollinearity angle is defined as the complement to $180^{\circ}$ of the 3 -dimensional angle between two vectors and the acoplanarity angle as the projection of the acollinearity angle onto a plane perpendicular to the beam direction.
} 
both $\tau^{ \pm}$leptons decay into a high-momentum neutrino and a low-momentum charged particle. This tail is adequately described by the $\mathrm{Z}^{0} \rightarrow \tau^{+} \tau^{-}$Monte Carlo. Only 3 events survive after the acoplanarity angle cut indicated in the figure.

One of the surviving events has a cone containing an electron pair from a photon conversion while the other cone is consistent with a 3 -prong $\tau^{-}$decay. Upon inspection, the event reveals the hits of a further low-momentum track of positive charge which is not reconstructed. This track is likely to be a positron from the decay of a $\tau^{+}$. The event is consistent with the $\mathrm{e}^{+} \mathbf{e}^{-} \rightarrow \tau^{+} \tau^{-} \gamma$ hypothesis and is removed by an algorithm, applied to all events, which identifies gamma conversions. The two remaining events are also consistent with the $\mathrm{e}^{+} \mathrm{e}^{-} \rightarrow \tau^{+} \tau^{-} \gamma$ hypothesis with the radiative photon included in one of the cones, raising the cone energy above the required threshold and giving rise to a large acoplanarity angle by modifying the cone axis. The expectation from the $\mathrm{Z}^{0} \rightarrow \ell^{+} \ell^{-}$Monte Carlo, after all cuts, of $1.0 \pm 0.5$ events (the error is statistical) is in agreement with the two observed events. Nevertheless, these two events are considered as $\mathrm{H}^{+} \mathrm{H}^{-}$candidates when deriving mass limits.

The detection efficiency for the process $\mathrm{H}^{+} \mathrm{H}^{-} \rightarrow\left(\tau^{+} \nu_{\tau}\right)\left(\tau^{-} \bar{\nu}_{\tau}\right)$ is $\approx 30 \%$ for $m_{\mathrm{H}^{ \pm}}>35 \mathrm{GeV}$. Systematic errors on the detection efficiency, mainly from neglecting the $\tau^{ \pm}$-polarization in the simulation, are small compared to the errors from Monte Carlo statistics (6\%). The inclusion of $\tau^{ \pm}$-polarization would tend to increase the detection efficiency. Assuming $B r\left(\mathrm{H}^{+} \rightarrow \tau^{+} \nu_{\tau}\right)=1$, a lower bound of $45.5 \mathrm{GeV}(95 \% \mathrm{CL})$ is obtained from this channel for the mass of the charged Higgs boson. The limit is calculated by comparing the expected number of signal events (Eq. 2), as a function of $m_{\mathrm{H}^{ \pm}}$, to the 95\% CL upper limit of 6.3 events (2 candidates) [19] for a possible signal. The expected number of signal events is, conservatively, decreased by $6 \%$ to take into account systematic errors.

\subsection{The semi-leptonic final state}

In the search for the semi-leptonic channel, $\mathrm{H}^{+} \mathrm{H}^{-} \rightarrow\left(\tau^{+} \nu_{\tau}\right)(\overline{\mathrm{c}} \mathrm{s})+(\mathrm{c} \overline{\mathrm{s}})\left(\tau^{-} \bar{\nu}_{\tau}\right)$, only the $\tau^{ \pm}$ decays with one charged track are admitted since decays of higher multiplicity are subject to a high background from $\mathrm{Z}^{0} \rightarrow$ hadrons. The final state is thus characterized by two hadronic jets, an isolated high-momentum charged track and missing energy. The analysis uses the sample of hadronic $\mathrm{Z}^{0}$ decays [11] which is then further reduced by requiring at least 7 tracks and 7 clusters. Remaining two-photon events and $Z^{0} \rightarrow q \bar{q} \gamma$ events with an energetic photon from initial state radiation are rejected by requiring that the forward energy flow ${ }^{2}$, measured in the forward calorimeters and in the endcap electromagnetic calorimeters, be less than 10 .

The feature of missing energy in the case of the Higgs boson signal is exploited by requiring that the scalar sum of all charged track momenta be less than $50 \mathrm{GeV}$ and that the energy in the electromagnetic and hadron calorimeters be less than $50 \mathrm{GeV}$ and $25 \mathrm{GeV}$, respectively. The total energy is then obtained by combining the energy deposited in the tracking chamber and in the electromagnetic and hadron calorimeters using an algorithm to globally correct the energy [20]. The algorithm gives an optimal weight to the energies measured in the tracking detector and in the calorimeters and, in the case of charged particles, reduces the effect from

\footnotetext{
${ }^{2}$ This quantity has been used in OPAL searches for the SM Higgs boson in the $\mathrm{e}^{+} \mathrm{e}^{-} \rightarrow \nu \bar{\nu} \mathrm{H}^{0}$ channel [12]. It is defined as $\left(E_{F}^{2}+E_{B}^{2}\right) / E_{t o t}^{2}$ where $E_{t o t}$ is the total energy of the event and $E_{F}$ and $E_{B}$ are weighted energies observed in the forward and backward cones defined by $|\cos \theta|>0.8$. The energies of tracks and clusters with polar angle $\theta$ are multiplied by $1 / \sin ^{2} \theta$, which gives more weight to tracks and clusters near the beam-pipe.
} 
double-counting of energy. The corrected energy is required to be between 30 and $75 \mathrm{GeV}$. The cuts described so far reduce the data sample to $\approx 5 \%$ of its original size.

The events are divided into two hemispheres by a plane perpendicular to the thrust axis. For each hemisphere the globally corrected momentum vector is obtained and the acollinearity and acoplanarity angles defined by the two hemisphere momentum vectors are calculated. To further reduce the background from hadronic $\mathrm{Z}^{0}$ decays, events are accepted if $\cos \theta_{\text {acol }}<0.95$ and $\cos \theta_{\text {acop }}<0.99$. For the remaining events the polar angle of the missing momentum vector is required to satisfy $\left|\cos \theta_{\text {miss }}\right|<0.94$ to further reduce two-photon and $q \bar{q} \gamma$ backgrounds. This selection provides an additional data suppression factor of $\approx 0.04$.

This selection is followed by a search for an isolated, energetic, charged track from a oneprong decay of the $\tau^{ \pm}$lepton. The charged track is required to have momentum between 3 and $15 \mathrm{GeV}$ and more than $7.5 \mathrm{GeV}$ energy (track and cluster energies together) is required to be in a narrow cone of $13^{\circ}$ half-angle around it. Isolation is imposed by allowing no other charged track in a wide cone of $30^{\circ}$ half-angle around the track and no more than $0.4 \mathrm{GeV}$ of calorimetric energy in the annulus between the wide and narrow cones. The isolation requirements yield a data suppression factor of $\approx 0.05$.

All tracks and clusters outside the wide isolation cone are assigned to the $\mathrm{H}^{+} \rightarrow \mathrm{cs}$ decay. The invariant mass, $m_{\mathrm{cs}}$, of this system is calculated. Since this search only addresses Higgs bosons with masses less than $M_{\mathrm{Z}^{0}} / 2, m_{\mathrm{cs}}<55 \mathrm{GeV}$ is required. Heavy Higgs bosons are produced with low kinetic energy, hence, the two hadron jets are typically in separate hemispheres and have an energy close to $m_{\mathrm{H}^{ \pm}} / 2$. The cs system is therefore divided in two jets using the plane perpendicular to its thrust axis. The globally corrected energies of the two jets are both required to be between 10 and $30 \mathrm{GeV}$.

A final selection cut is applied in the scatter plot of $m_{\mathrm{cs}}$ vs. $\cos \theta_{\mathrm{cs}}$, shown in Fig. 2, where $\theta_{\mathrm{cs}}$ is the acollinearity angle of the two jet momenta. The figure shows the distribution for three simulated $\mathrm{H}^{+} \mathrm{H}^{-}$event samples with $m_{\mathrm{H}^{ \pm}}=36,40$ and $45 \mathrm{GeV}$. The correlation expected from the decay kinematics is clearly visible. Events with mass close to the kinematic limit $\left(m_{\mathrm{H}^{ \pm}}=45 \mathrm{GeV}\right)$ cluster at $\cos \theta_{\mathrm{cs}} \approx 1$ while those with $m_{\mathrm{H}^{ \pm}}=36 \mathrm{GeV}$ cluster at $\cos \theta_{\mathrm{cs}}<0.4$. The final selection is indicated by the two parallel lines. Before this cut there remain 5 events in the data sample $\left(\approx 1.5 \times 10^{-6}\right.$ of the original number of events $)$ which are indicated by the large dots. Only one event, with $m_{\mathrm{cs}} \approx 34 \mathrm{GeV}$ and $\cos \theta_{\mathrm{cs}} \approx 0.2$, satisfies the selection. This event is taken as a Higgs boson candidate when deriving mass limits. The same analysis, applied to a sample of 3.5 million simulated hadronic $\mathrm{Z}^{0}$ decays leaves 4 events before the final cut and also one single event after.

The detection efficiencies for the $\mathrm{H}^{+} \mathrm{H}^{-}$signal in the semi-leptonic final state vary between $6 \%$ and $10 \%$ in the mass range $35 \mathrm{GeV}<m_{\mathrm{H}^{ \pm}}<46 \mathrm{GeV}$. The statistical error of these values is less than $10 \%$. The systematic error on the detection efficiencies, mainly from modelling fragmentation, is estimated to be $5 \%$ from comparing simulated event samples with various sets of fragmentation parameters. As in the leptonic channel, the inclusion of $\tau^{ \pm}$polarization in the simulation would tend to increase the signal detection efficiency. Assuming $B r\left(\mathrm{H}^{+} \rightarrow\right.$ $\left.\tau^{+} \nu_{\tau}\right)=0.5$, a lower bound of $45.0 \mathrm{GeV}(95 \% \mathrm{CL})$ is obtained from this channel for the mass of the charged Higgs boson. The limit is calculated by comparing the expected number of signal events (Eq. 2), as a function of $m_{\mathrm{H}^{ \pm}}$, to the 95\% CL upper limit of 4.74 events (1 candidate) [19] for a possible signal. The expected number of signal events is decreased by $11 \%$ to take into account systematic errors. 


\subsection{The hadronic final state}

The hadronic channel, $\mathrm{H}^{+} \mathrm{H}^{-} \rightarrow(c \bar{s})(\overline{\mathrm{c}} \mathrm{s})$, is characterized by an event topology with four hadron jets. The predominant background is from higher order QCD processes, $Z^{0} \rightarrow q \bar{q} g g$ and $q \bar{q} q \bar{q}$. Candidate events are selected by requiring visible energy, $E_{v i s}$, larger than $40 \mathrm{GeV}$. This cut, together with the requirement that the distribution of the electromagnetic cluster energy be longitudinally balanced, $\left|\Sigma\left(E_{i} \cos \theta_{i}\right)\right| / \Sigma E_{i}<0.65$ (the sum runs over all clusters, $E_{i}$ and $\theta_{i}$ are the energy and polar angle of cluster $i$ ), eliminates two-photon events and $Z^{0} \rightarrow q \bar{q} \gamma$ events.

Jets are defined by the Durham scheme [21] of the YCLUS jet finder [22] using the visible energy as the scale parameter and fixing the jet resolution parameter, $y_{\text {cut }}$, to 0.01 . The jet finder is applied to the charged tracks and to those electromagnetic clusters which have no track pointing to them. Events with at least 4 jets are selected. If more than 4 jets are found in an event, the jet finder is reapplied with the value of $y_{\text {cut }}$ increased until the number of reconstructed jets is exactly 4 . The sphericity of the event is required to be larger than 0.3 . Fig. 3 shows the distribution of the number of jets (a) and the sphericity distribution (b) for the data, for a sample of 3.5 million simulated $\mathrm{Z}^{0}$ hadronic decays and for a simulated $\mathrm{H}^{+} \mathrm{H}^{-}$ signal sample with $m_{\mathrm{H}^{ \pm}}=42 \mathrm{GeV}$. After these cuts the data sample is reduced to $2.5 \%$ of its original size. The selection efficiency of the $(\bar{c} \bar{s})(\bar{c} s)$ final state varies between $55 \%$ and $61 \%$ for $m_{\mathrm{H}^{ \pm}}$between 36 and $45 \mathrm{GeV}$.

A kinematic fit [23] is applied to the remaining events assuming the $\mathrm{H}^{+} \mathrm{H}^{-} \rightarrow(\mathrm{c} \overline{\mathrm{s}})(\overline{\mathrm{c}} \mathrm{s})$ decay hypothesis. The four jets are associated in pairs to the $\mathrm{H}^{+}$and $\mathrm{H}^{-}$bosons. The inputs to the fit are the jet four-momenta together with their estimated measurement errors. The total energy and momentum of the events are constrained to those of the colliding $\mathrm{e}^{+} \mathrm{e}^{-}$beams (the effect of initial state radiation is neglected) and the invariant mass of the two jet pairs is required to be equal $\left(m_{\mathrm{H}^{+}}=m_{\mathrm{H}^{-}}\right)$. The kinematic fit is applied to the 3 possible associations and the one yielding the smallest $\chi^{2}$ is retained. The smallest $\chi^{2}$ is required to be less than 45 for 5 degrees of freedom. The invariant mass distribution of the jet pairs obtained in this manner is shown in Fig. 3 (c). The observed small difference in shape between the data and the sample of simulated hadronic $\mathrm{Z}^{0}$ decays can be explained by known inaccuracies in modelling the phase space distributions of events with more than three partons [24]. In addition, uncertainties in describing fragmentation also contribute. The possible effect of an overall calibration error of the mass scale is estimated by shifting the mass of the Monte Carlo events and evaluating the overlap with the data. The best overlap is obtained for a shift of $-100 \mathrm{MeV}$. The invariant mass distribution for a simulated $\mathrm{H}^{+} \mathrm{H}^{-}$signal, also shown in Fig. 3 (c), has a narrow peak close to the nominal Higgs boson mass and a broad component due to incorrect jet associations and measurement errors.

In order to identify a possible Higgs boson signal, the mass distribution of the data is searched for a statistically significant local excess in the form of a narrow peak. Both the data and the signal distributions for different $m_{\mathrm{H}^{ \pm}}$are parametrized. The distribution of the data between 30 and $45 \mathrm{GeV}$ is well described by a third order polynomial. The signal distribution is described by the superposition of a third order polynomial and a Gaussian of width $\sigma$ centred at $M_{0}$. The fractional area of the signal contained in the Gaussian part is denoted by $f$. The shape of the non-Gaussian part is fairly independent of the Higgs boson mass. Values of these parameters are obtained from fitting simultaneously the invariant mass spectra of six signal samples with masses between 36 and $45 \mathrm{GeV}$. In the fit the third order polynomial is constrained to be the same for all masses. 
To search for a Higgs boson signal, the invariant mass distribution of the data is scanned in steps of $0.25 \mathrm{GeV}$ in the mass range from 35 to $44.5 \mathrm{GeV}$. At each point of the scan:

- the parameters $M_{0}, \sigma$ and $f$ are determined by linear interpolation between the set of discrete values obtained for the six fixed Higgs boson masses;

- a third order polynomial is fitted to the data in the range 30 to $45 \mathrm{GeV}$ excluding the mass window given by $M_{0} \pm 1.64 \sigma$;

- the fitted function is subtracted from the data distribution and a Gaussian with parameters $M_{0}$ and $\sigma$ is fitted to the distribution of the difference. It is assumed that the broad component of the signal is absorbed in the polynomial describing the data. The area of the Gaussian, $\alpha$, is determined;

- the $95 \%$ CL upper limit for a possible Higgs boson signal is determined from

$$
N_{\max }=\frac{\alpha}{\epsilon f}+1.64 \sigma\left(\frac{\alpha}{\epsilon f}\right)
$$

where $\epsilon$ is the detection efficiency for the $\mathrm{H}^{+} \mathrm{H}^{-}$signal and $\sigma\left(\frac{\alpha}{\epsilon f}\right)$ is the error on $\frac{\alpha}{\epsilon f}$. The latter is obtained by propagating the errors of the detection efficiency and those of $\alpha$ and $f$ given by the fit.

The systematic error on the detection efficiency arises mainly from modelling the hadronization and fragmentation processes. It is estimated to be less than $5 \%$ from the deviation in normalization of the data and the sample of simulated hadronic $\mathrm{Z}^{0}$ decays after selection. The error from Monte Carlo statistics is $3 \%$. Thus, an overall error of $6 \%$ is assigned to the detection efficiency. The systematic error from the determination of $\alpha$ is found to be small in comparison.

Assuming a branching fraction $B r\left(\mathrm{H}^{+} \rightarrow \tau^{+} \nu_{\tau}\right)=0$, a lower bound of $44.2 \mathrm{GeV}$ (95\% CL) is obtained from this channel for the mass of the charged Higgs boson. The limit is calculated by comparing the expected number of signal events (Eq. 2), as a function of $m_{\mathrm{H}^{ \pm}}$, to the $95 \%$ CL upper limit using Eq. 3.

If the scale of the invariant mass of the Higgs boson signal were shifted by $-100 \mathrm{MeV}$, as suggested by the small discrepancy between the distributions for the data and for the sample of simulated hadronic $\mathrm{Z}^{0}$ decays, this limit would change to $44.3 \mathrm{GeV}$. The stability of the result is further checked by varying the cut in $\chi^{2}$ which is used to select the correct jet association. The corresponding variations of the mass bounds are smaller than $\pm 100 \mathrm{MeV}$.

\section{Results}

The lower bounds for the mass of the charged Higgs boson, at the 95\% CL, obtained from the searches in the leptonic, semi-leptonic and hadronic channels, are presented in Fig. 4 as a function of the branching ratio $B r\left(\mathrm{H}^{+} \rightarrow \tau^{+} \nu_{\tau}\right)$. The limits are obtained using the tree level expression given by Eq. 1 for the process $\mathrm{Z}^{0} \rightarrow \mathrm{H}^{+} \mathrm{H}^{-}$. They take into account the integrated luminosities of the data distributed over $\sqrt{s}$ and the selection efficiencies as a function of $m_{\mathrm{H}^{ \pm}}$ with their statistical and systematic errors. The effect of initial-state radiation on the $\mathrm{Z}^{0}$ 


\begin{tabular}{||l||c|c|c|c|c|c|c||}
\hline$B r\left(\mathrm{H}^{+} \rightarrow \tau^{+} \nu_{\tau}\right):$ & 0.0 & $\mathbf{0 . 0 5}$ & $\mathbf{0 . 2}$ & $\mathbf{0 . 4}$ & $\mathbf{0 . 6}$ & $\mathbf{0 . 8}$ & 1.0 \\
\hline$m_{\mathrm{H}^{ \pm}}[\mathrm{GeV}]:$ & 44.2 & 44.1 & 44.7 & 45.0 & 45.2 & 45.4 & 45.5 \\
\hline
\end{tabular}

Table 1: Lower limits for $m_{\mathrm{H}^{ \pm}}$, valid at the $95 \% \mathrm{CL}$, as a function of the branching ratio $\operatorname{Br}\left(\mathrm{H}^{+} \rightarrow \tau^{+} \nu_{\tau}\right)$.

line shape is taken into account by using Eq. 2 to calculate the number of expected $\mathrm{H}^{+} \mathrm{H}^{-}$ events. The shift of the mass limits, less than $100 \mathrm{MeV}$, from the reduction of the effective centre of mass energy due to initial-state radiation is also included. The two events remaining in the leptonic final state and the single event remaining in the semi-leptonic final state are considered as possible Higgs boson candidates. The global exclusion, shown in the figure by the full line, is the envelope of the three individual limits. A more sophisticated and less conservative statistical method to combine the results from the three channels would lead to a marginal improvement only. Close to the kinematical limit the cross section falls rapidly with increasing mass (Eq. 1), and therefore the limit is weakly affected by changes of the detection efficiency (e.g. by combining channels). The precise mass values of the limit are listed in Table 1 for selected values of the branching ratio $B r\left(\mathrm{H}^{+} \rightarrow \tau^{+} \nu_{\tau}\right)$. The highest value of $m_{\mathrm{H}^{ \pm}}$ which is excluded at the $95 \% \mathrm{CL}$ independent of the branching ratios is $44.1 \mathrm{GeV}$. This bound is obtained assuming that the channels $\mathrm{H}^{+} \rightarrow \tau^{+} \nu_{\tau}$ and $\mathrm{H}^{+} \rightarrow c \overline{\mathrm{s}}$ saturate the decay of the charged Higgs boson but is also valid in the case of sizeable contributions from $\mathrm{H}^{+} \rightarrow c \bar{b}$. The quoted results improve the mass bounds obtained previously by OPAL [6] and by other experiments [7] [8].

\section{Acknowledgements}

It is a pleasure to thank the SL Division for the efficient operation of the LEP accelerator, the precise information on the absolute energy, and their continuing close cooperation with our experimental group. In addition to the support staff at our own institutions we are pleased to acknowledge the

Department of Energy, USA,

National Science Foundation, USA,

Particle Physics and Astronomy Research Council, UK,

Natural Sciences and Engineering Research Council, Canada,

Fussefeld Foundation,

Israel Ministry of Science,

Israel Science Foundation, administered by the Israel Academy of Science and Humanities,

Minerva Gesellschaft,

Japanese Ministry of Education, Science and Culture (the Monbusho) and a grant under the Monbusho International Science Research Program,

German Israeli Bi-national Science Foundation (GIF),

Direction des Sciences de la Matière du Commissariat à l'Energie Atomique, France,

Bundesministerium für Forschung und Technologie, Germany,

National Research Council of Canada,

Hungarian Foundation for Scientific Research, OTKA T-016660 and OTKA F-015089. 


\section{References}

[1] S. Weinberg, Phys. Rev. Lett. 19 (1967) 1264;

A. Salam, Proceedings of the 8th Nobel Symposium edited by N. Svartholm (Almquist and Wiksell, Stockholm, 1968), p. 367;

S. Glashow, Nucl. Phys. B22 (1961) 579.

[2] P. W. Higgs, Phys. Lett. 12 (1964) 132; Phys. Rev. Lett. 13 (1964) 508; Phys. Rev. 145 (1966) 1156.

[3] J. F. Gunion, H. E. Haber, G. Kane and S. Dawson, The Higgs Hunter's Guide (AddisonWesley, 1990).

[4] ALEPH Collaboration, D. Buskulic et al., Phys. Lett. B313 (1993) 299;

DELPHI Collaboration, P. Abreu et al., Nucl. Phys. B421 (1994) 3;

L3 Collaboration, O. Adriani et al., Phys. Lett. B303 (1993) 391;

OPAL Collaboration, R. Akers et al., Phys. Lett. B327 (1994) 397.

[5] ALEPH Collaboration, D. Buskulic et al., Phys. Lett. B313 (1993) 312;

DELPHI Collaboration, P. Abreu et al., Z. Phys. C64 (1994) 183;

L3 Collaboration, O. Adriani et al., Phys. Lett. B294 (1992) 457;

OPAL Collaboration, R. Akers et al., Z. Phys. C64 (1994) 1.

[6] OPAL Collaboration, M.Z. Akrawy et al., Phys. Lett. B242 (1990) 299.

[7] DELPHI Collaboration, P. Abreu et al., Z. Phys. C64 (1994) 183.

[8] ALEPH Collaboration, D. Decamp et al., Prog. Phys. Lett. 216 (1992) 253;

L3 Collaboration, O. Adriani et al., Phys. Lett. B294 (1992) 457.

[9] P.J. Franzini et al., in Z Physics at LEP 1, CERN 89-08, Vol. 2, p. 59.

[10] OPAL Collaboration, K. Ahmet et al., Nucl. Instrum. Methods A305 (1991) 275.

[11] OPAL Collaboration, G. Alexander et al., Z. Phys. C52 (1991) 175.

[12] OPAL Collaboration, R. Akers et al., Phys. Lett. B327 (1994) 397.

[13] PYTHIA 5.7, T. Sjöstrand, Comput. Phys. Commun. 82 (1994) 74;

T. Sjöstrand, CERN-TH/7112/93 (1993, revised August 1994).

[14] JETSET 7.3, T. Sjöstrand, Comput. Phys. Commun. 39 (1986) 347;

T. Sjöstrand and M. Bengston, Comput. Phys. Commun. 43 (1987) 367;

H.-U. Bengston and T. Sjöstrand, Comput. Phys. Commun. 46 (1987) 43;

T. Sjöstrand, CERN-TH/6488/92.

[15] OPAL Collaboration, M. Z. Akrawy et al., Z. Phys. C47 (1990) 505;

OPAL Collaboration, P. D. Acton et al., Z. Phys. C58 (1993) 387.

[16] KORALZ 4.0 Monte Carlo, S. Jadach, B. F. L. Ward and Z. Wąs, Comput. Phys. Commun. 79 (1994) 503 . 
[17] BABAMC Monte Carlo, M. Böhm, A. Denner and W. Hollik, Nucl. Phys. B304 (1988) 687;

F. A. Berends, R. Kleiss and W. Hollik, Nucl. Phys. B304 (1988) 712.

[18] J. Allison et al., Nucl. Instrum. Methods A317 (1992) 47.

[19] Review of Particle Properties, Section on Statistics, Phys. Rev. D50 (1994) 1280.

[20] OPAL Collaboration, M. Z. Akrawy et al., Phys. Lett. B253 (1991) 511.

[21] S. Catani, Yu. L. Dokshitzer, M. Olsson, G. Turnock and B. R. Webber, Phys. Lett. B269 (1991) 432.

[22] S. Bethke, QCD - 20 Years Later, Preprint, Heidelberg University, HD-PY 92/12.

[23] A. G. Frodesen, O. Skjeggestad and H. Tøfte, Probability and Statistics in Particle Physics, (Universitetsforlaget, Oslo, 1979), Section 10.8, p. 308.

[24] OPAL Collaboration, R. Akers et al., Z. Phys. C65 (1995) 367. 


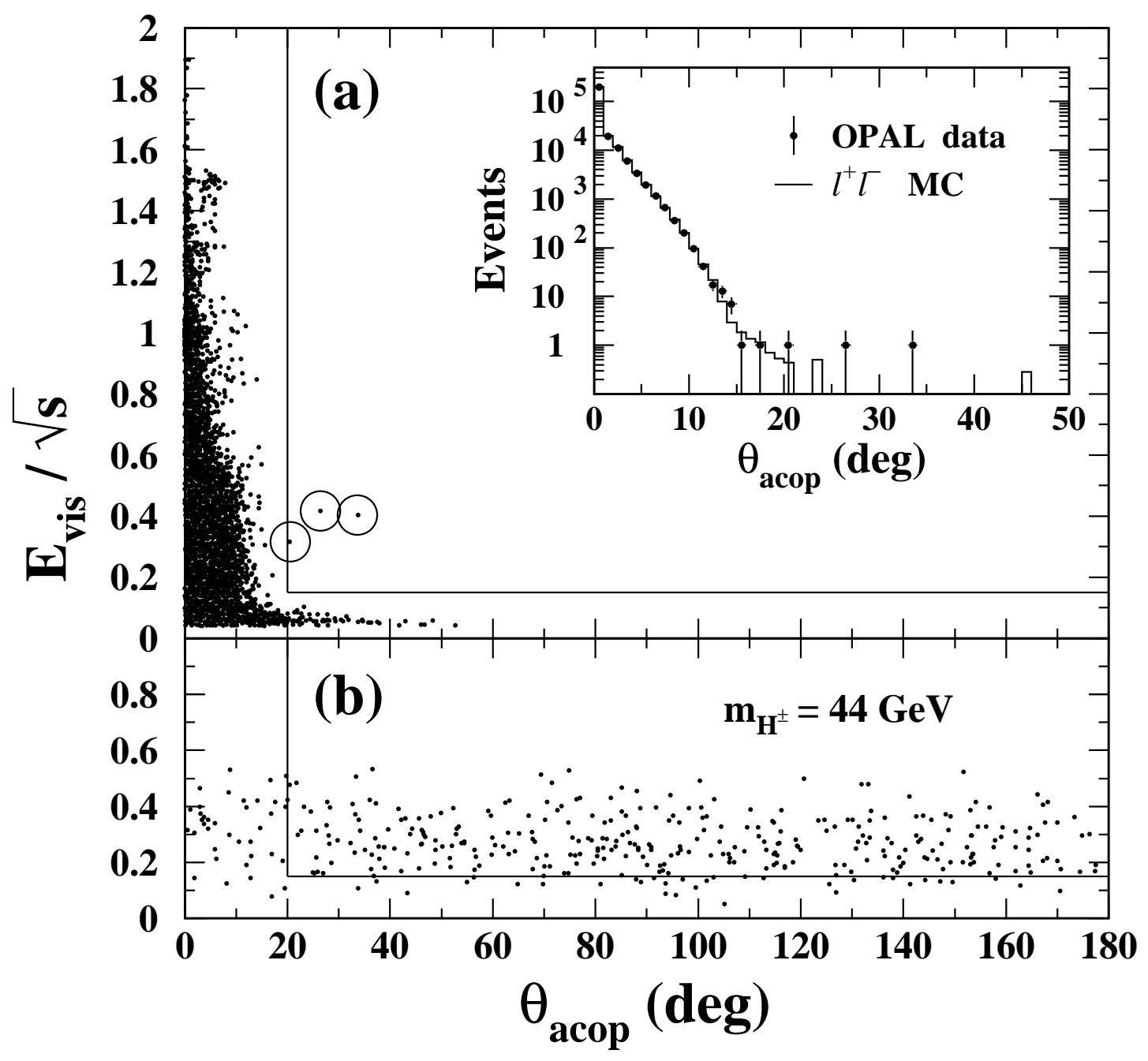

Figure 1: Scatter plot of the visible energy vs. the acoplanarity angle, defined by the two cone axes, (a) for the data and (b) for a simulated $\mathrm{H}^{+} \mathrm{H}^{-}$sample with $m_{\mathrm{H}^{ \pm}}=44 \mathrm{GeV}$. The cuts on the visible energy and the acoplanarity angle are indicated. The 3 data events that survived these cuts are circled. The insert shows the acoplanarity angle distribution of the data (points with error bars) compared to that of a sample of simulated $\mathrm{Z}^{0} \rightarrow \ell^{+} \ell^{-}$events (histogram), after the cut on the visible energy. 


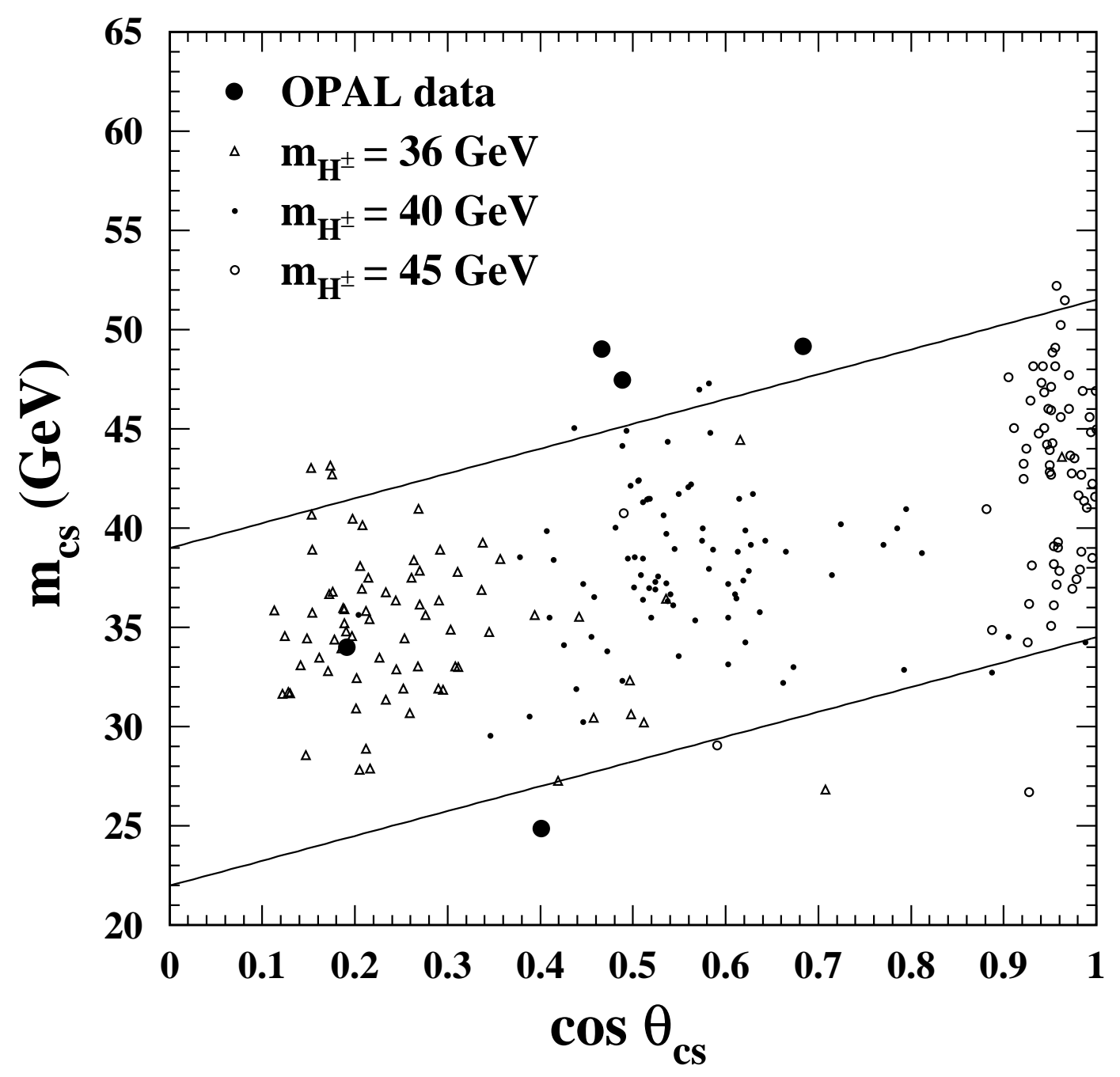

Figure 2: Scatter plot of the invariant mass, $m_{\mathrm{cs}}$, vs. the acollinearity angle of the two hemisphere momentum vectors, for three simulated $\mathrm{H}^{+} \mathrm{H}^{-}$samples with $m_{\mathrm{H}^{ \pm}}=36,40$ and $45 \mathrm{GeV}$. The large dots indicate the 5 data events which remain before the selection indicated by the parallel lines. 

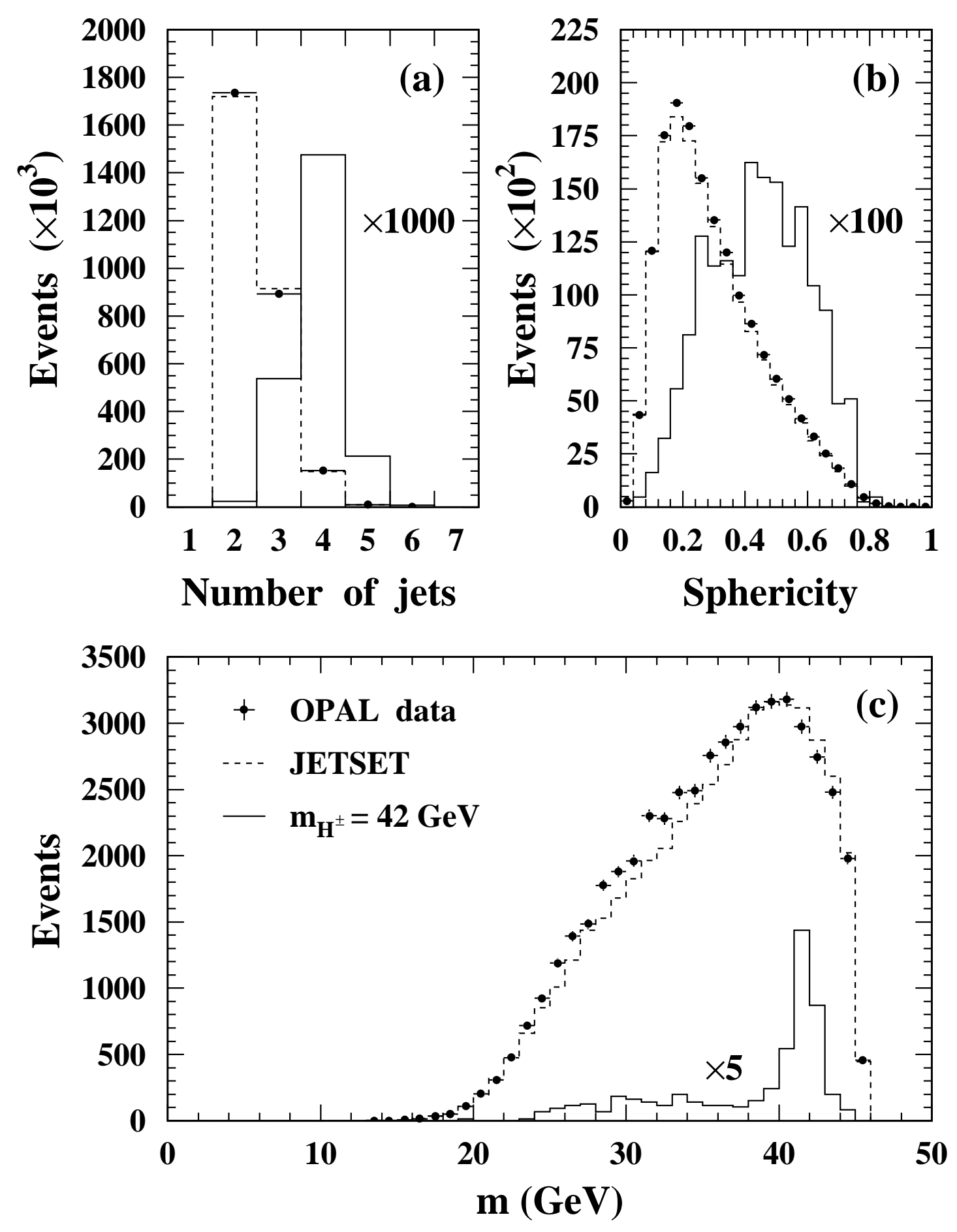

Figure 3: (a) Distribution of the number of jets, (b) sphericity distribution after the 4-jet requirement and (c) invariant mass distribution obtained by the constrained kinematical fit after all cuts, for the data (points with error bars), for a sample of simulated hadronic $\mathrm{Z}^{0}$ decays (dashed histogram) and for a simulated $\mathrm{H}^{+} \mathrm{H}^{-}$sample with $m_{\mathrm{H}^{ \pm}}=42 \mathrm{GeV}$ (full-line histogram). The distributions are normalized to the data before the selection and the signal distribution is scaled up by the factors indicated, assuming $B r\left(\mathrm{H}^{+} \rightarrow \tau^{+} \nu_{\tau}\right)=0$. 


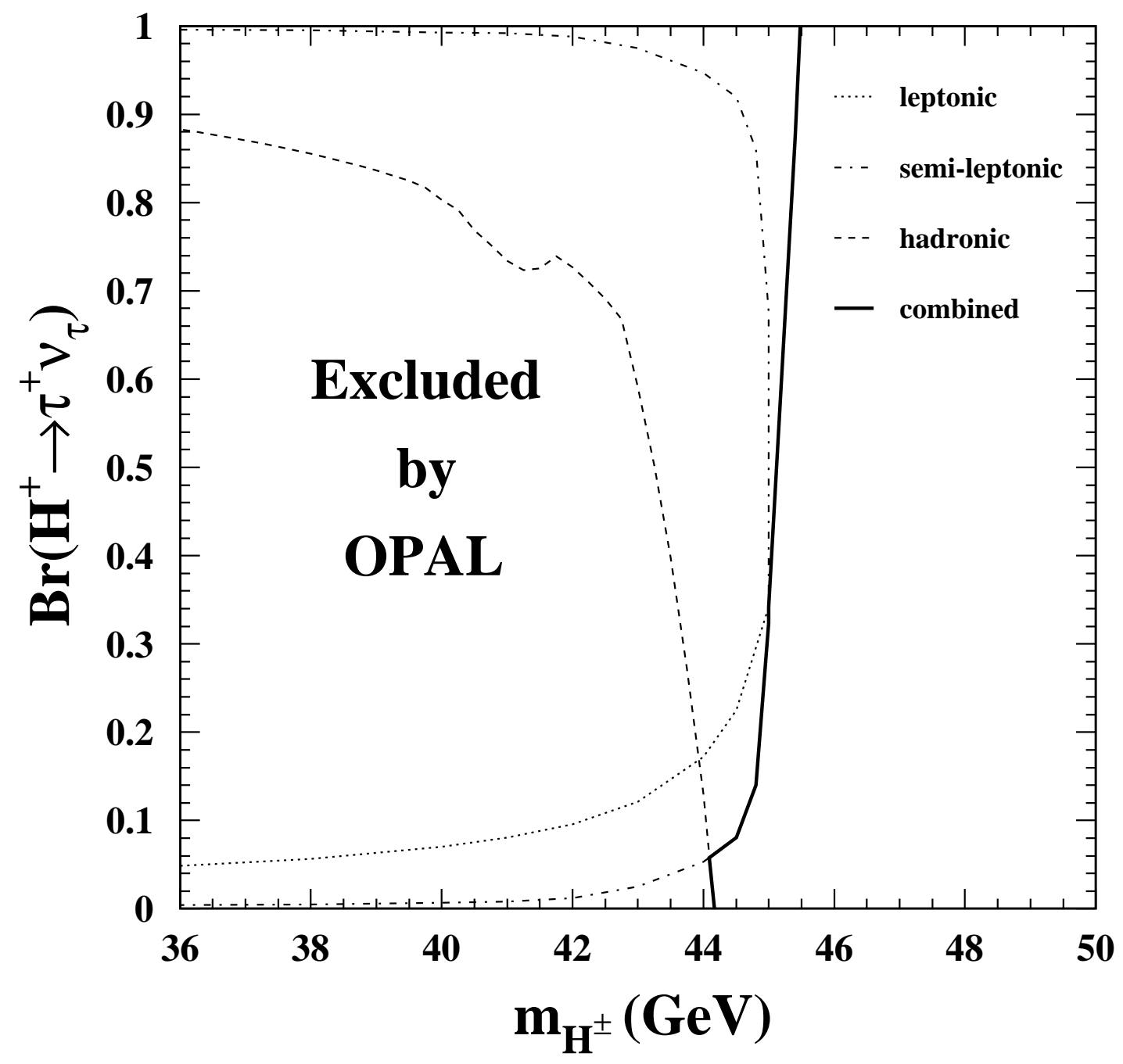

Figure 4: Regions in the $B r\left(\mathrm{H}^{+} \rightarrow \tau^{+} \nu_{\tau}\right)$ vs. $m_{\mathrm{H}^{ \pm}}$plane which are excluded at the $95 \%$ CL by the searches in the leptonic channel (dotted line), in the semi-leptonic channel (dash-dotted line) and in the hadronic channel (dashed line). The combined exclusion limit is indicated by the full line. 